\title{
Re-operation of idiopathic macular hole after failed initial surgery
}

\author{
Libor Hejsek, Jaroslava Dusova, Alexandr Stepanov, Pavel Rozsival
}

Background. Persistent idiopathic macular hole (IMH) after initial pars plana vitrectomy (PPV) appears in approx. $12 \%$ of cases. It is, therefore, one of the most common complications. IMH re-operation results tend to be better in eyes where IMH had been closed, while IMH re-operations in cases when the initial surgery had failed tend to be less successful: IMH often becomes even larger and visual acuity decreases, anatomical success rate of closure is about $60 \%$ and the resulting postoperative visual acuity is about 20/100.

Aims. To establish anatomical and functional success rate of repeated operations of recurrent idiopathic macular hole. Materials and Methods. We evaluated the retrospective results of re-operations of persisting IMH that is cases in which the initial surgery did not result in closure: 6 eyes in 6 patients out of the overall 55 operated on IMH (primary PPV failed in almost $11 \%$ of cases). The follow-up period was 18 months (since the first surgery).

Results and Conclusion. After the second PPV, the IMH was closed in $100 \%$ of operated eyes. It was not necessary to perform a third PPV during the follow-up period. In all cases the visual acuity improved. Anatomical and functional results of IMH treatment can be considered satisfactory. We consider the following combination of factors significant for the anatomical success rate of PPV: IMH size and stage, technical precision of the ILM peeling and the suction of the IMH contents, strict adherence to the recommended head positioning in early postoperative period, and IMH duration.

Key words: recurrent macular holes, re-operation, pars plana vitrectomy

Received: June 24, 2013; Accepted with revision: November 18, 2013; Available online: November 29, 2013 http://dx.doi.org/10.5507/bp.2013.088

Department of Ophthalmology, Faculty of Medicine in Hradec Kralove, Charles University in Prague and University Hospital Hradec Kralove, Hradec Kralove, Czech Republic

Corresponding author: Libor Hejsek, e-mail: libor.hejsek@fnhk.cz

\section{INTRODUCTION}

Meta-analysis of 1654 eyes treated with various modifications of pars plana vitrectomy (PPV) describes $88 \%$ success rate of the idiopathic macular hole (IMH) surgery. Therefore IMH persistence after PPV remains one of the most common complications, other complications are, for instance, developing cataracts or retinal detachment ${ }^{1}$.

In previous studies the authors have shown anatomical and functional success of repeated operations of recurrent IMH. Late reopening of IMH primarily closed after surgery is described in $5-10 \%$ of cases. A third reopen-

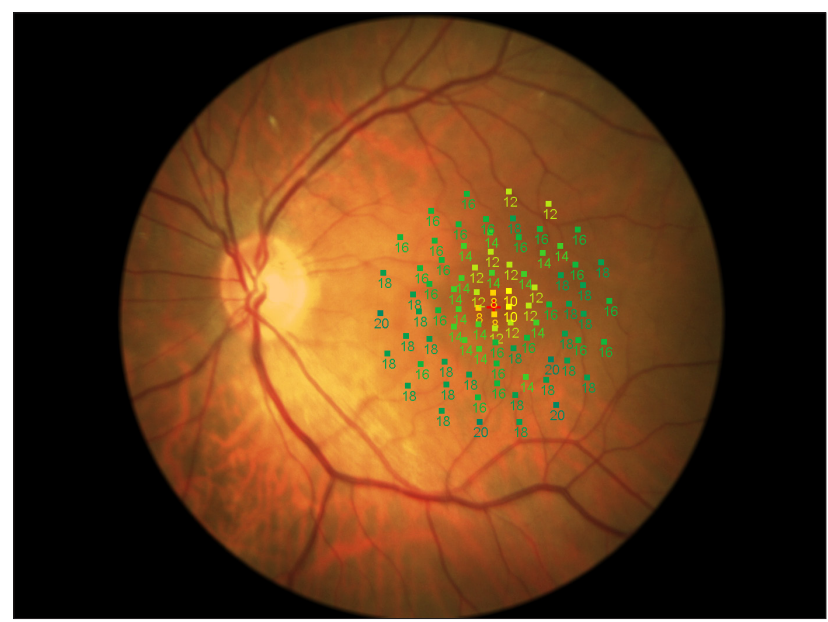

Fig. 1. Microperimetry before IMH surgery. ing of IMH twice previously closed is rarely described. The results of repeated IMH surgery are better in eyes in which IMH had previously been closed ${ }^{2}$. By contrast, the results of IMH re-operation in cases in which the initial surgery was not successful (IMH often enlarges even more and the visual acuity decreases) have anatomical success rate of about $60 \%$ and final postoperative visual acuity of 20/100 (ref. $\left.{ }^{3}\right)$.

An example of functional deterioration when IMH didn't close after the first PPV is illustrated on microperimetry (Fig. 1 and 2). (ref. $\left.{ }^{4}\right)$.

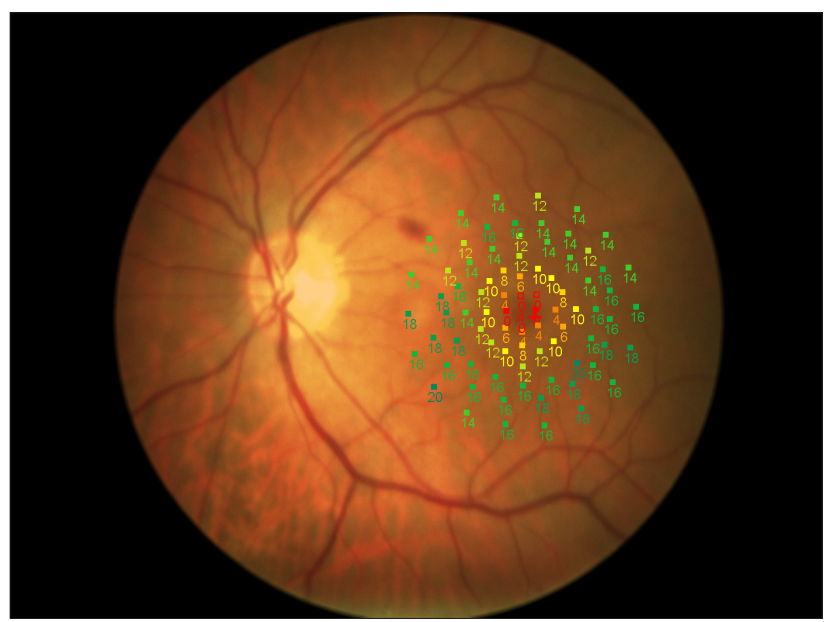

Fig. 2. Microperimetry after IMH surgery. 
The pathophysiology of initial surgery failure or reopening of IMH is not entirely clear; it may be related to insufficient stability of the glial plug in the IMH center. Surgery alone may also have some other effect such as glial upregulation ${ }^{5}$. Insufficient function of retinal pigment epithelium (RPE), cystoid macular edema (CME), cataract surgery or development of epiretinal membrane (ERM) may contribute to a recurrence of a closed IMH $\left(\right.$ ref. $\left.^{6,7}\right)$.

\section{MATERIALS AND METHODS}

In our set we evaluate the results of persistent IMH re-operations, i.e. the cases when the initial surgery did not result in closure. This is the retrospective consecutive case-series study. Slit-lamp biomicroscopy and OCT were used for anatomical evaluation. Best-corrected visual acuity (BCVA) measured on ETDRS optotypes was used to evaluate function. The set consisted of 6 eyes in 6 patients ( 5 women, 1 man), out of the total number of 55 patients operated on IMH (the initial surgery failed in almost $11 \%$ of cases). The follow-up period was 18 months (since the first surgery). All the patients underwent a second PPV 5-11 ( 7.8 average) months after the initial surgery. In all cases but one, ILM was peeled in an area of 2-3 disc diameters during the primary PPV. Air (in 1 case), SF6 (in 1 case) and $\mathrm{C} 3 \mathrm{~F} 8$ (in 4 cases) were used for tamponade during the initial surgery. During the second surgery, enlargement of the ILM peeling area was performed with Eckardt microforceps after it had been stained with a blue dye (MembraneBlue Dual ${ }^{\circledR}$ ), and the elevated IMH edge was loosened manually with a silicone brush. In the final stage of the surgery the IMH contents were suctioned and a temporary air tamponade was put in place. In all cases, gas tamponade with perfluoropropane $(\mathrm{C} 3 \mathrm{~F} 8)$ was used at the end of the surgery and face-down positioning was recommended for 7 days. The non-parametric Wilcoxon paired-sample test was used for result evaluation.

\section{RESULTS}

The distribution of IMH stages (at the examination before the initial surgery) according to Gass classification was as follows: 4 cases of stage 3 IMH and 2 cases of stage 4 IMH. None of the primary surgeries on stage 2 IMH demanded re-operation.

The initial visual acuity (before primary PPV) ranged from 20/50 to 20/200, average 20/100 (median 20/100). In all cases the visual acuity after the second PPV improved in comparison with the initial visual acuity (before the primary PPV). The best-corrected visual acuity was improved to 20/32 - 20/160, average 20/60 (median $20 / 80)$. This improvement was statistically significant $(P=0.02)$.

After the second PPV, IMH was closed in $100 \%$ of cases. A third PPV was not necessary during the follow-up period. In half of the cases ( 3 out of 6 ) it was necessary to perform also a complicated cataract surgery (during

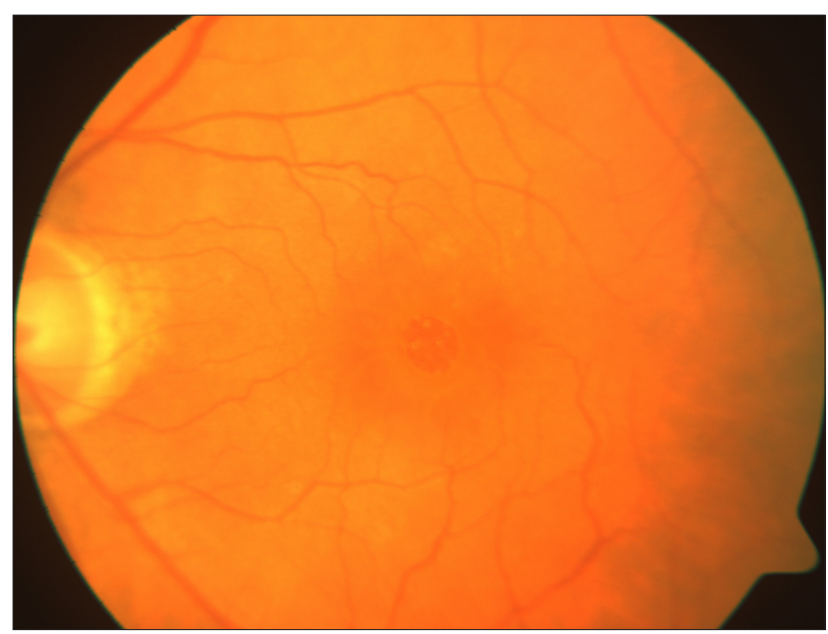

Fig. 3. Primary IMH.

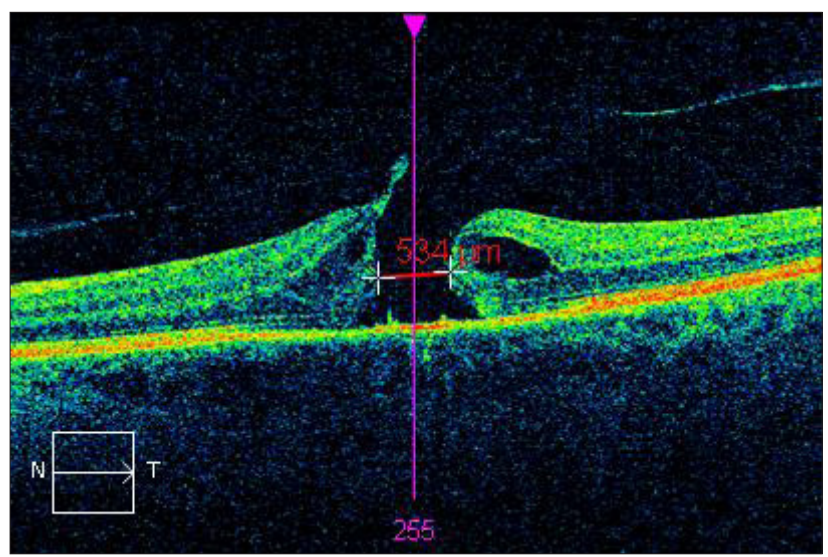

Fig. 4. Diameter of IMH was $534 \mu \mathrm{m}$.

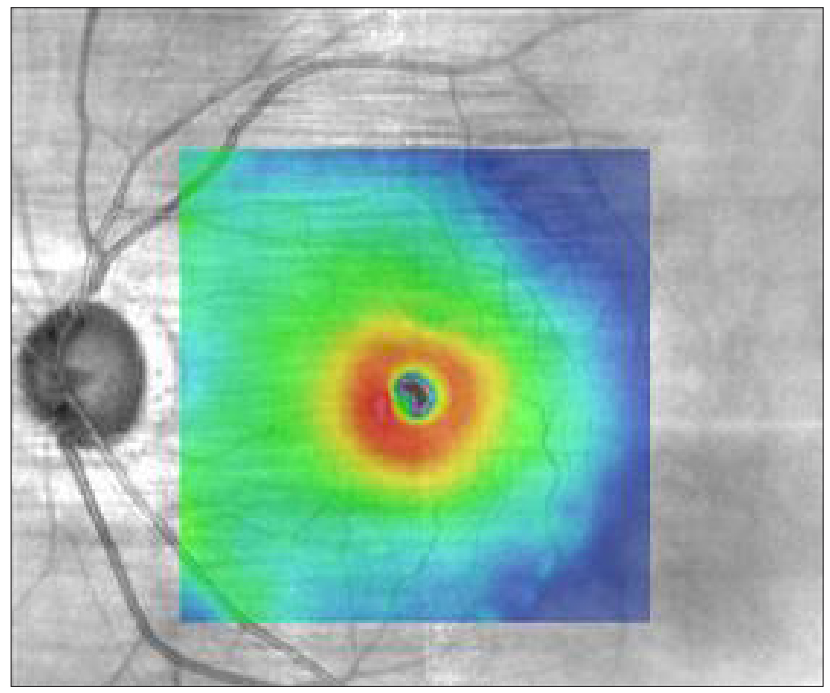

Fig. 5. Edematic center.

the second PPV (phacoemulsification with intraocular lens implantation). We didn't observe any development of retinal detachment during the postoperative period.

An example of the progress of the disease during the observation period is illustrated in Figs. 3-10. Primary IMH (Fig. 3) was in stage 3 according to Gass classifi- 


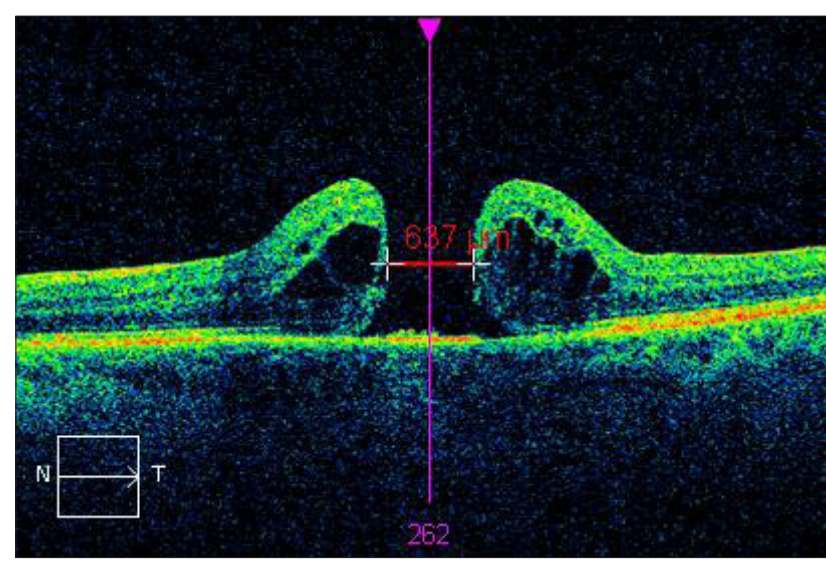

Fig. 6. IMH diameter grew to $637 \mu \mathrm{m}$.

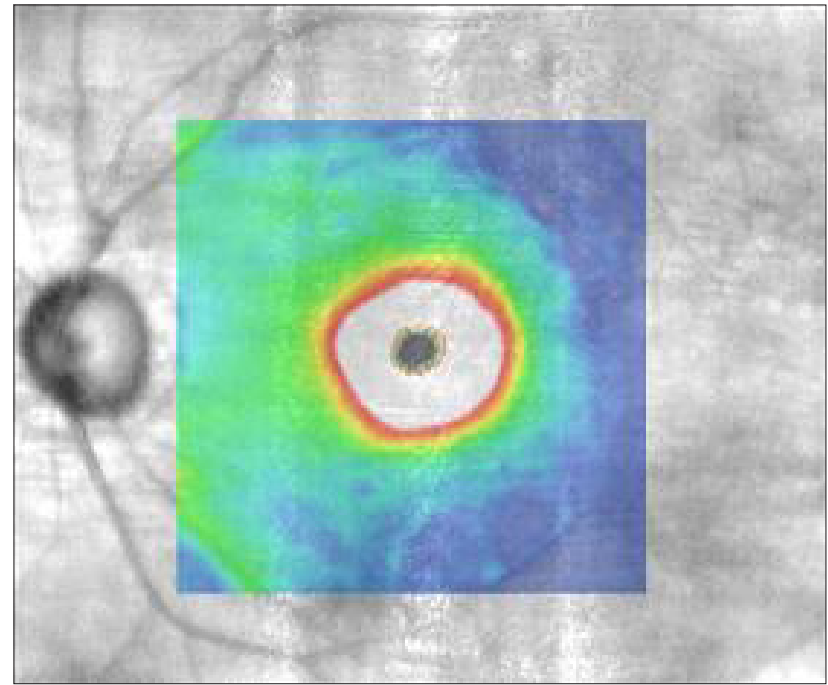

Fig. 7. Cystic changes around the hole.

cation, with the visual acuity of $20 / 100$, the minimum diameter of IMH was $534 \mu \mathrm{m}$ (Fig. 4) and the center was edematic (Fig. 5).

The condition has deteriorated both objectively and subjectively after the initial surgery. The visual acuity decreased to 20/200, the IMH diameter grew to $637 \mu \mathrm{m}$ (Fig. 6) and cystic changes around the hole have progressed (Fig. 7).

The second PPV was performed after 5 months, in this case together with a cataract surgery. IMH closed completely (Fig. 8 and 9), visual acuity improved to 20/80 and the cystoid macular edema has disappeared (Fig. 10).

\section{DISCUSSION}

Despite high success rate of macular hole surgery, unclosed IMH after primary PPV is still one of the most frequent complications ${ }^{8}$. There is not much information available about persistent macular holes. Most of the information came in the early 1990's, when Kelly and Wendel introduced a surgical technigue for IMH.

The first results of re-operation for IMH after the unsuccessful first PPV (with ILM peeling) has been de-

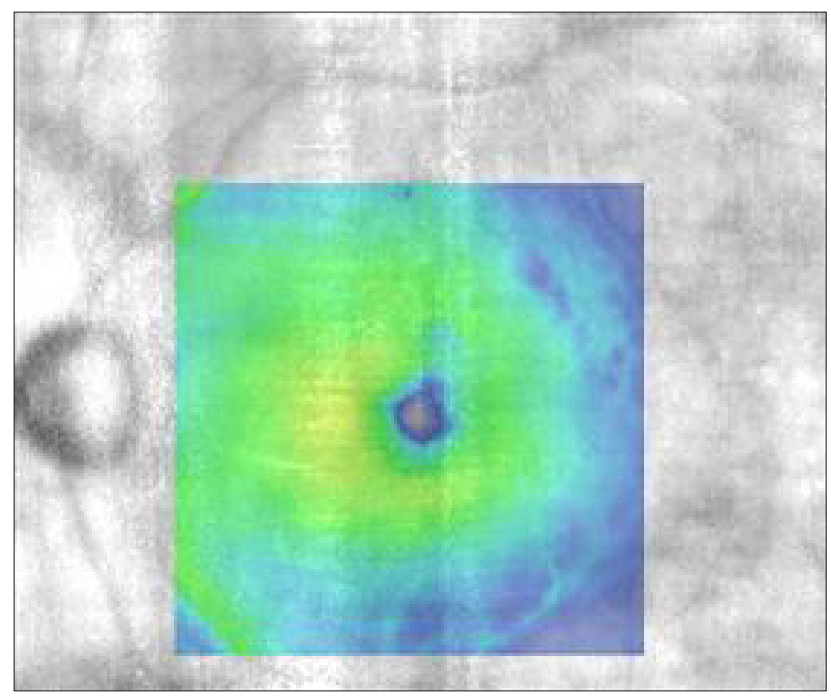

Fig. 8. IMH closed completely.

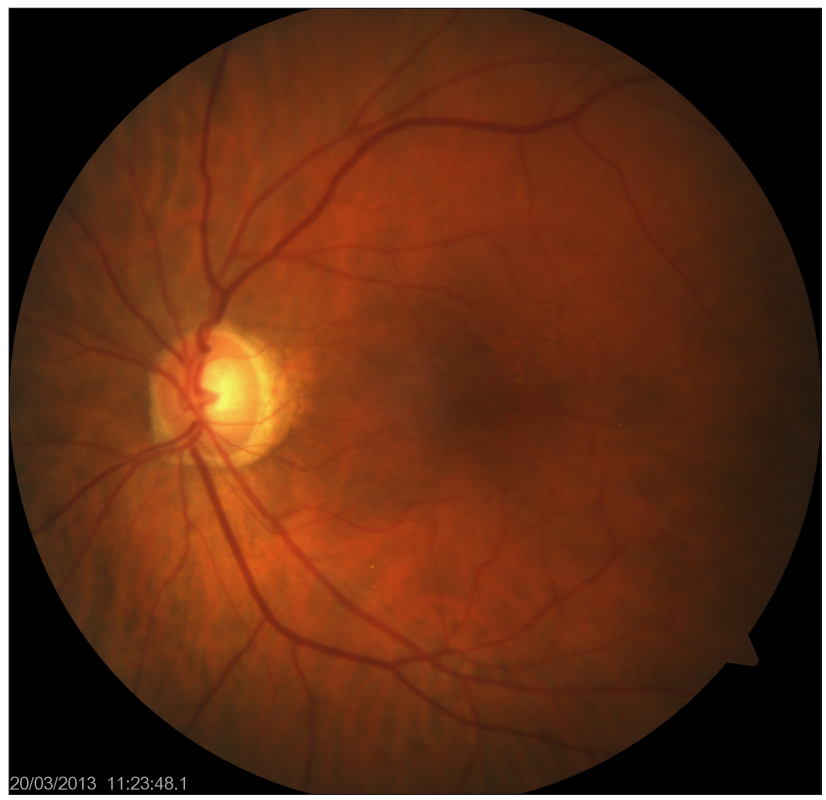

Fig. 9. OCT IMH closed completelyl.

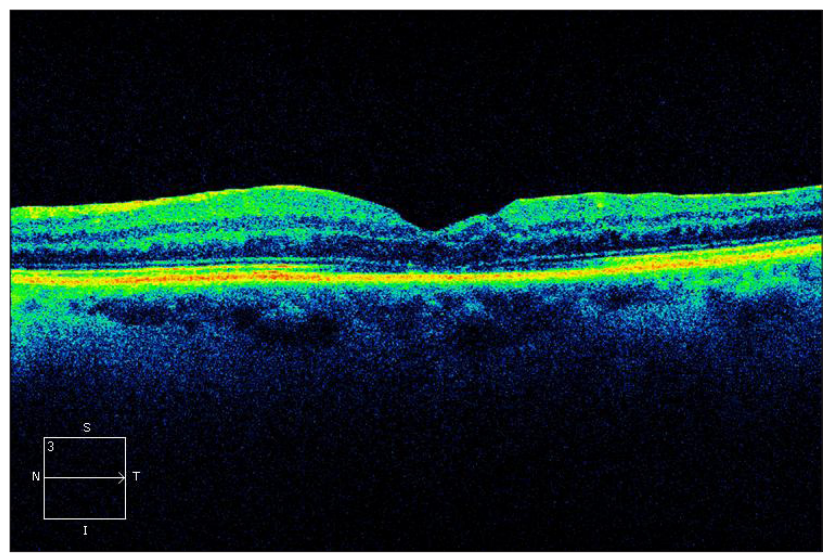

Fig. 10. Cystoid macular. edema has disappeared. 
scribed in papers including relatively small numbers of patients. Da Mata et al. published IMH closure in 2 of 3 cases after second PPV with silicon oil ${ }^{9}$. Rizzo et al. achived success of reoperation using "heavy silicon oil" $\left(\right.$ ref. $\left.{ }^{10}\right)$. This type of tamponade was effective even in the upright position.

Hillenkamp et al. performed a retrospective study of 28 unclosed IMH ( ref. $^{11}$ ). The rate of anatomical closure was $68 \%$ after the second surgery. There was found the correlation of IMH preoperative configuration (according to OCT) and efficacy of reoperation (PPV without enlargement of the peeled ILM area). An prominent cuff of subretinal fluid at the margin of the IMH was a strong prognostic factor for anatomical closure and also BCVA improvement. This result was explained as a lack of adhesion between macular hole margin and retinal pigment epitelium, which may facilitate closure of IMH. Higher rate of anatomical closure reported Valldeparas et al. Retrospective study involved reoperation of 52 unclosed IMH with $76 \%$ success. ILM peeling during the initial and the second PPV was carried out only in selected cases $^{12}$.

D'Souza et al. followed 30 reoperated cases $^{13}$. Anatomical closure was achieved in 52\%. Reoperation surgical technique involved PPV with enlargement of the ILM rhexis and gas tamponade. This methodology was relatively less efficient compared to report of Valldeparas et al. We can assume, that re-PPV with enlargement of the peeling area is more likely to succeed in macular holes whose ILM have already been peeled.

We consider the following combination of factors significant for the anatomical success rate of primary PPV: IMH size and stage, technical precision of the ILM peeling (to relief all possible tractions and to prevent further epimacular membrane formation) and the suction of the IMH contents, strict adherence to the recommended head positioning in early postoperative period, and a sufficient amount of gas tamponade.

Repeated PPV's and gas tamponade contribute to the development of complicated cataract which can limit postoperative functional results ${ }^{14}$. Simultaneous cataract and PPV surgery is technically possible.

We understand the unusually high closure percentage in re-operated IMH to be the result of our manual loosening of the elevated and often rigid edges of IMH with a silicone brush. IMH contents were suctioned at the end of the surgery and the liquid was completely substituted with a temporary air tamponade. We always observed a significant IMH diameter decrease at the end of this move. Surgical technique was in all cases constant, but number of patients is relatively low.

In our set of cases, the time factor does not seem overly significant in the process of planning IMH re-operation (anatomical and functional results are positive even in case of a longer interval between the primary and secondary PPV), nevertheless, we lean towards the opinion that a relatively short interval between the surgeries is advisable.

\section{CONCLUSION}

IMH was closed in $100 \%$ of eyes after the second PPV. A third PPV was not necessary during the follow-up period. Best-corrected visual acuity was improved in all the re-operated cases. We, therefore, consider the anatomical and functional results of IMH treatment satisfactory. But due to limited sample size of patients and absence of control group it is not possible to determine retreatment strategy or select any subgroup with persistent macular holes, where the benefit of subsequent surgery is highest.

Reducing the number of failed primary surgeries remains a challenge.

\section{ACKNOWLEDGEMENT}

Authorship contributions: All authors contributed equally to preparing the manuscript.

Conflict of interest statement: The authors stated that there are no conflicts of interest regarding the publication of this article.

\section{REFERENCES}

1. Mester V, Kuhn F. Internal limiting membrane removal in the management of full thickness macular holes. Am J Ophthalmol 2000;129(6):769-77.

2. Smiddy WE, Sjaarda RN, Glaser BM, Flynn HW Jr, Thompson JT, Hanham A, Murphy RP. Reoperation after failed macular hole surgery. Retina 1996;16:13-8.

3. Thompson JT, Sjaarda RN. Results of macular hole surgery in patients over 80 years of age. Retina 2000;20:433-8.

4. Hejsek L. Mikroperimetrie a její klinický prínos při onemocnění sítnice. Česká a slovenská oftalmologie 2006;62(6):423-6.

5. Jackson TL, Hillenkamp J, Williamson TH, Clarke KW, Almubarak Al, Marshall J. An experimental model of rhegmatogenous retinal detachment: surgical results and glial cell response. Invest Ophthalmol Vis Sci 2003:44:4026-34.

6. Funata M, Wendel RT, de la Cruz Z, Green WR. Clinicopathologic study of bilateral macular holes treated with pars plana vitrectomy and gas tamponade. Retina 1992;12:289-98.

7. Paques $M$, Massin $P$, Blain $P$, Duquesnoy AS, Gaudric A. Longterm incidence of reopening of macular holes. Ophthalmology 2000;107:760-5.

8. Mester V, Kuhn F. Internal limiting membrane removal in the management of full thickness macular holes. Am J Ophthalmol 2000;129:769-77.

9. Da Mata AP, Burk SE, Foster RE, Riemann CD, Petersen MR, Nehemy Má, Augsburger JJ. Long-term follow-up of indocyanine greenassisted peeling of the retinal internal limiting membrane during vitrectomy surgery for idiopathic macular hole repair. Journal Ophthalmol 2004;111(12):2246-53.

10. Rizzo S, Belting C, Genovesi-Ebert F, Cresti F, Vento A, Martini R. Successful treatment of persistent macular holes using "heavy silicone oil" as intraocular tamponade. Retina 2006;26(8):905-8.

11. Hillenkamp J, Kraus J, Framme C, Jackson TL, Roider J, Gabel VP, Sachs HG. Retreatment of full-thickness macular hole: predictive value of optical coherence tomography. Br J Ophthalmol 2007;91(11):1445-9.

12. Valldeperas $X$, Wong D. Is It Worth Reoperating on Macular Holes? Ophthalmology 2008;115(1):158-63.

13. D'Souza M, Chaudhary V, Devenyi R, Kertes PJ, Lam WC. Re-operation of idiopathic full-thickness macular holes after initial surgery with internal limiting membrane peel. Br J Ophthalmol 2011;95(11):1564-7.

14. Niwa $T$, Terasaki $H$, Kondo $M$, Piao $C H$, Suzuki T, Miyake $Y$. Function and morphology of macula before and after removal of idiopathic epiretinal membrane. Invest Ophthalmol Vis Sci 2003;44:1652-6. 